Washington University School of Medicine Digital Commons@Becker

Open Access Publications

$3-1-2020$

\title{
Antibody-mediated rejection after lung transplantation
}

Amit I. Bery

Washington University School of Medicine in St. Louis

Ramsey R. Hachem

Washington University School of Medicine in St. Louis

Follow this and additional works at: https://digitalcommons.wustl.edu/open_access_pubs Please let us know how this document benefits you.

\section{Recommended Citation}

Bery, Amit I. and Hachem, Ramsey R., "Antibody-mediated rejection after lung transplantation." Annals of Translational Medicine. 8, 6. 411 (2020).

https://digitalcommons.wustl.edu/open_access_pubs/9873

This Open Access Publication is brought to you for free and open access by Digital Commons@Becker. It has been accepted for inclusion in Open Access Publications by an authorized administrator of Digital Commons@Becker. For more information, please contact vanam@wustl.edu. 


\title{
Antibody-mediated rejection after lung transplantation
}

\author{
Amit I. Bery, Ramsey R. Hachem \\ Division of Pulmonary \& Critical Care, Washington University School of Medicine, Saint Louis, MO, USA \\ Contributions: (I) Conception and design: All authors; (II) Administrative support: RR Hachem; (III) Provision of study materials or patients: None; (IV) \\ Collection and assembly of data: None; (V) Data analysis and interpretation: All authors; (VI) Manuscript writing: All authors; (VII) Final approval of \\ manuscript: All authors. \\ Correspondence to: Amit I. Bery, MD. Division of Pulmonary and Critical Care Medicine, Washington University School of Medicine, 660 S Euclid \\ Avenue, Campus Box 8052, Saint Louis, MO 63108, USA. Email: amit.bery@wustl.edu.
}

\begin{abstract}
Antibody-mediated rejection (AMR) has been identified as a significant form of acute allograft dysfunction in lung transplantation. The development of consensus diagnostic criteria has created a uniform definition of AMR; however, significant limitations of these criteria have been identified. Treatment modalities for AMR have been adapted from other areas of medicine and data on the effectiveness of these therapies in AMR are limited. AMR is often refractory to these therapies, and graft failure and death are common. AMR is associated with increased rates of chronic lung allograft dysfunction (CLAD) and poor long-term survival. In this review, we discuss the history of AMR and describe known mechanisms, application of the consensus diagnostic criteria, data for current treatment strategies, and long-term outcomes. In addition, we highlight current gaps in knowledge, ongoing research, and future directions to address these gaps. Promising diagnostic techniques are actively being investigated that may allow for early detection and treatment of AMR. We conclude that further investigation is required to identify and define chronic and subclinical AMR, and head-to-head comparisons of currently used treatment protocols are necessary to identify an optimal treatment approach. Gaps in knowledge regarding the epidemiology, mechanisms, diagnosis, and treatment of AMR continue to exist and future research should focus on these aspects.
\end{abstract}

Keywords: Lung transplantation; graft rejection; allograft tolerance; acute lung injury; complement component; complement C4d; diagnostic techniques and procedures; plasmapheresis; intravenous immunoglobulins (IVIG); rituximab; eculizumab; outcome assessment

Submitted Jul 21, 2019. Accepted for publication Nov 08, 2019.

doi: 10.21037/atm.2019.11.86

View this article at: http://dx.doi.org/10.21037/atm.2019.11.86

\section{Introduction}

Lung transplantation is the ultimate treatment for selected patients with end-stage lung disease. While advances in surgical technique have improved early survival, long term outcomes remain disappointing, and the median survival after transplantation in the most recent era is 6.5 years (1). Chronic lung allograft dysfunction (CLAD) is the leading cause of death beyond the first year after lung transplantation and has emerged as the main barrier to better long-term outcomes (1). Antibody-mediated rejection (AMR) has been increasingly recognized after lung transplantation and has been consistently identified as a significant cause of morbidity, CLAD, and graft failure $(2-7)$. Here, we present a review of our current understanding of AMR and discuss ongoing research and future directions to further our understanding of AMR and improve the management of this serious complication after lung transplantation.

\section{History}

Our understanding of AMR in lung transplantation was historically limited to hyperacute rejection, which 
occurs when preformed donor-specific antibodies (DSA) bind to mismatched human leukocyte antigens (HLA) $(8,9)$. Patients developed significant and often fatal graft failure intra-operatively or in the immediate postoperative period with hemorrhagic pulmonary edema and diffuse pulmonary infiltrates on imaging studies $(8,10,11)$. Severe graft dysfunction occurred in spite of intensive immunosuppression targeting T-cell proliferation and activity, which suggested a role for humoral immunity in precipitating this response. Typically, these patients were found to have DSA and a positive direct lymphocytotoxicity crossmatch. Further investigation found that allosensitization was associated with worse survival after transplantation unless the reactive HLA were avoided in a prospective donor $(8-10,12,13)$. Case reports of hyperacute rejection suggested the need for standardization of pretransplant crossmatching $(6,8)$. Although hyperacute rejection has become rare in the current era because of improved HLA antibody detection assays, it illustrates that HLA antibodies can cause fulminant graft failure and that the capillary endothelium is the focal point of injury.

Over time, cases occurring later after transplantation were recognized and our understanding of acute AMR began to evolve. Badesch and colleagues presented a case series of 5 patients with pulmonary capillaritis ranging from 3 weeks to months after transplant (14). In this series, all patients presented with alveolar hemorrhage and graft failure with temporary improvement in graft function with intensification of immunosuppression and plasmapheresis (14). They postulated a humoral immunologic response, though testing for DSA or antibody binding on pathologic specimens was not performed (14). Magro and colleagues described the histologic features of 22 lung transplant recipients who developed pulmonary capillaritis ranging from 1 to 33 months after transplantation (15). They described septal capillary necrosis with staining positive for complement deposition (C1q, C3, and $\mathrm{C} 4 \mathrm{~d}$ ) and immunoglobulin G (IgG) (15). All patients were tested and found to be negative for panel-reactive antibodies (PRA) both before transplantation and at the time of acute rejection; however, the use of older and less sensitive assays limits the significance of this finding $(11,15)$. Treatment with plasmapheresis showed improvement in graft function and a decrease in post-pheresis capillary injury and complement deposition (15). Witt and colleagues detailed a series of 21 patients with acute AMR (3). In this series, all patients had clinical allograft dysfunction, DSA, histology of acute lung injury, and capillary endothelial C4d deposition (3). Six patients (29\%) died of refractory AMR, while 15 survived to hospital discharge (3). One patient had a diagnosis of bronchiolitis obliterans syndrome (BOS) at AMR diagnosis, and 13 of the remaining 14 survivors developed CLAD during follow up (3). Overall 15/21 (71\%) patients died during the study period and median survival after diagnosis of AMR was 593 days (3). These studies established the basis for further investigation of the diagnosis and management of acute AMR.

\section{Pathogenesis}

Mechanisms of AMR were initially elucidated by investigations in transplantation of other solid organs, especially in kidney transplantation $(16,17)$. This work has shown hyperacute, acute, and chronic forms of AMR in which activation of allospecific B-cells and plasma cells leads to formation of DSA that bind to HLA on the endothelium of vessels within the allograft $(6,16,18,19)$. Antibody biding leads to complement dependent and independent recruitment of immune cells leading to graft dysfunction and tissue injury $(6,16,18-20)$.

DSA were first identified in renal transplant recipients in the 1960s and were postulated to be the underlying cause for immediate graft failure. Subsequent investigation confirmed that the presence of preformed antibodies in recipient sera was associated with a high rate of graft failure. These failures led to the use of a crossmatch of the recipients serum with donor cells and assays to identify DSA (21-23). Since that time, highly sensitive and specific solid phase assays for the identification of HLA antibodies have been developed $(6,16,24)$. Recipients may have preexisting HLA antibodies (as a result of prior sensitizing events such as blood transfusion, pregnancy, or previous organ transplantation) or may develop HLA antibodies de novo after transplantation $(6,9,11,12,17)$. Recipients may have antibodies to MHC class I antigens (HLA-A, HLA-B, or HLA-C) which are present on all nucleated cells, or to MHC class II antigens (HLA-DQ, HLA-DR, HLA-DP) which are present on antigen presenting cells (APCs) (25). Notably, pro-inflammatory cytokines have been shown to induce the expression of class II HLA on pulmonary endothelial cells $(26,27)$. Recipients may also develop antibodies to non-HLA antigens (allo- or auto-antigens); however, no highly sensitive assays have been developed to detect such antibodies to date $(5,25,28)$.

Complement-binding DSA are associated with worse 
outcomes in kidney and heart transplant recipients, and preliminary work echoes these findings in lung transplantation $(3,29,30)$. DSA bind antigens on donor endothelial cells with activation of the classical complement cascade and formation of the membrane attack complex (MAC). The MAC causes endothelial cell injury with exposure of the basement membrane and activation of the coagulation cascade leading to thrombosis and infarction. Moreover, complement factors $3 \mathrm{a}$ and $5 \mathrm{a}$ (C3a and C5a) are chemokines that attract immune cells to the allograft propagating inflammation and graft injury $(11,15,31)$. Importantly, not all DSA bind and activate complement, and DSA-associated complement-independent mechanisms of allograft injury have been studied in vitro and in vivo models of solid organ transplantation (6,16,31-36). Proposed mechanisms include activation of signaling cascades that lead to endothelial and smooth muscle cell proliferation, release of inflammatory cytokines and chemokines, and von Willebrand factor (vWF) and P-selectin mediated platelet activation $(6,11,31-36)$. These findings not only implicate DSA in complement-independent mechanisms of AMR, but also suggest a role for DSA in chronic allograft rejection $(6,11,31,36,37)$.

Recent work by Li and colleagues has shown that the immunopathology of AMR may be different in lung allografts compared to allografts of other solid organs (38). This group utilized a mouse lung retransplantation model and found that tolerant pulmonary allografts developed bronchus-associated lymphoid tissue (BALT) (38). Suppression of FoxP $3^{+}$regulatory T-cells in this model led to the absence of BALT formation and development of AMR (38). These findings suggest that regulatory T-cells residing in BALT of pulmonary allografts suppress B-cell activation locally and that antigen presentation can occur within the allograft (38). These findings are contrary to models of AMR in other solid organ transplants where humoral responses are regulated peripherally (38). This is further echoed by the differences noted in the diagnosis, management, and outcomes of AMR in lung allografts as compared to other solid organ transplants $(3-6,31,39,40)$.

\section{Clinical manifestations and diagnosis}

The International Society for Heart and Lung Transplantation (ISHLT) convened a working group in 2016 to create a uniform definition of AMR (6). The definition was largely based on experience in kidney and heart transplantation. According to this definition, a definite diagnosis of AMR requires the presence of allograft dysfunction, DSA, characteristic lung pathology, complement factor $4 \mathrm{~d}(\mathrm{C} 4 \mathrm{~d})$ deposition on capillary endothelium, and the exclusion of alternate etiologies of graft dysfunction (6). The working group recognized potential shortcomings of these diagnostic criteria and proposed a qualitative assessment of the certainty of the diagnosis as being definite, probable, or possible based on the number of criteria met (6). Graft dysfunction associated with clinical AMR may range from fulminant respiratory failure to asymptomatic dysfunction identified on surveillance spirometry (6). Chronic AMR is a defined form of rejection in kidney transplantation and has been suggested in both heart and lung transplantation, but there is no specific definition in lung transplantation $(16,17)$. Although investigations in lung transplantation have shown an association between DSA and CLAD, which most commonly presents as BOS, it is unclear if such cases represent chronic AMR as these are typically indistinguishable from cases that lack DSA $(2-4,6,9,11,37,39,41)$. Furthermore, depletion of DSA has been associated with greater freedom from BOS (37). Roux and colleagues reviewed 206 transplanted patients and found that restrictive allograft syndrome (RAS) was only present in patients who had DSA and developed AMR, while BOS or mixed CLAD (BOS and RAS) were found in all groups of patients regardless of presence of DSA or AMR (5). It is appealing to consider whether RAS is a form of chronic AMR, but definitive data have been lacking to date. The ISHLT working group also recognized sub-clinical AMR as the detection of histologic criteria of AMR in the absence of graft dysfunction (6). Chronic and sub-clinical AMR remain poorly described in lung transplantation and further investigation is required to identify the significance of these diagnoses.

The presence of DSA is requisite for a diagnosis of AMR based on these criteria; however, only a minority of patients with DSA go on to develop AMR $(11,16,42)$. Furthermore, detection of DSA can be missed due to phasic release or absence of the antigen on the screening assay (6). Highly sensitive solid phase assays for DSA provide mean fluorescence intensity (MFI) values which do not always accurately depict antibody strength (43). Specificity can be improved with assays for binding of complement factor $1 \mathrm{q}$ (C1q) but this may decrease sensitivity (43).

Multiple pathologic findings of AMR have been described in lung allografts including capillary 
inflammation, endothelialitis, and acute lung injury $(9,44,45)$. The earliest report of hyperacute AMR of a lung allograft noted the presence of acute lung injury with hyaline membrane formation along with alveolar edema and hemorrhage (8). Subsequent reports described pulmonary capillaritis and neutrophilic infiltration as the hallmark findings of pulmonary AMR (46). However, further investigation has shown that neutrophils are just one of the many cell types that are part of the spectrum of microvascular inflammation and the absence of neutrophil infiltration or capillaritis should not rule out AMR $(31,47,48)$. Neutrophilic capillaritis is nonspecific and has been associated with graft dysfunction in the absence of AMR $(41,42,48)$. Furthermore, the presence of capillaritis may be obscured by severe acute lung injury. Acute lung injury may present as alveolar edema, alveolar hemorrhage, hyaline membrane formation with diffuse alveolar damage, fibrin deposition, intravascular platelet or fibrin thrombi, or arteriolar fibrinoid necrosis $(2,47,49)$. These findings suggest that the histopathologic findings of AMR are nonspecific and underscore the need for a multidisciplinary approach to diagnosis.

Staining for complement split product $\mathrm{C} 4 \mathrm{~d}$ is the most controversial criterion for the diagnosis of AMR (50). Complement split products bind to endothelial cells noncovalently and are subsequently inactivated by membrane cofactor protein, decay accelerating factor, and CD59 (51). However, unlike other complement split products, C4d binds the endothelium covalently via a thioester bond (51). This covalent bond is stable, resistant to shedding, and allows $\mathrm{C} 4 \mathrm{~d}$ to be detected long after other complement split products have been inactivated (51). While C4d staining gained popularity in diagnosis of AMR in renal allografts and provides direct evidence of the immunepathologic effect of antibodies, it has proven to be a challenging criterion in AMR of lung transplant recipients $(5,6,11,44)$. C4d staining has been shown to be positive in pathology other than AMR such as ischemia-reperfusion injury, high grade ACR, and infection $(5,6)$. Furthermore, C4d has been shown to be positive in only a minority of cases of AMR in lung transplant recipients, giving rise to "C4d-negative" AMR (2-4,52). It remains unclear if C4dnegative AMR represents a unique form of AMR due to complement-independent pathways or if this is related to limitations of staining or interpretation of specimens (52). Finally, Roden and colleagues found poor correlation between immunofluorescence and immunohistochemical testing for $\mathrm{C} 4 \mathrm{~d}$, and poor inter-reader reliability among pathologists (50).

\section{Treatment}

Treatment options for AMR in lung transplantation have been adapted from other areas of medicine. Data supporting the use of individual interventions are sparse and no randomized clinical trials or head-to-head comparisons have been undertaken, making it difficult to draw conclusions about the efficacy of any single intervention (Table 1). Treatment regimens generally consist of multiple interventions and are individualized based on clinical course and response to other therapies.

\section{Corticosteroids}

Corticosteroids inhibit the early steps of the acute inflammatory response by altering the transcription of numerous genes in leukocytes (53). These genes affect leukocyte maturation, differentiation, distribution, and trafficking (53). High-dose corticosteroids are utilized in treatment regimens for AMR across kidney, heart, and lung transplantation $(4,16,54)$. Astor and colleagues presented a retrospective review of 40 patients of whom only $43 \%$ responded to steroid monotherapy (46). Steroid resistance was identified as a marker for AMR in early reports $(14,46)$.

\section{Plasmapheresis}

Plasmapheresis separates and discards plasma from whole blood replacing it with fresh frozen plasma or albumin and, therefore, eliminates circulating antibodies. In addition to reducing DSA, plasmapheresis has been shown to decrease deposition of complement split products (15). While plasmapheresis depletes circulating antibody, it does not decrease antibody production and can result in a rebound increase in antibody production. Due to its ability to eliminate circulating antibody, plasmapheresis gained popularity for the treatment of AMR in kidney transplantation (55). Retrospective studies in AMR of lung allografts have found variable benefit with use of plasmapheresis $(4,7,8,10,12,14,15,46,56)$. These results are challenging to interpret, as plasmapheresis is rarely used as monotherapy and plasmapheresis protocols differ among studies.

\section{Intravenous immunoglobulin (IVIG)}

IVIG has traditionally been used in the treatment of 
Table 1 Treatment modalities for AMR in lung transplantation

\begin{tabular}{lll}
\hline Treatment & Mechanism & References \\
\hline Corticosteroids & Alter transcription of genes in leukocytes & $(4,14,16,46,53,54)$ \\
Plasmapheresis & Elimination of circulating antibodies; decrease deposition of & $(4,7,8,10,12,14,15,46,55,56)$ \\
& complement split products & $(4,57-59)$ \\
Intravenous Immunoglobulin & Unclear; proposed mechanisms include modulation of Fc $\gamma$ receptor, \\
(IVIG) & inactivation of DSA, downregulation of B-cells, inhibition of & $(37,60)$ \\
Rituximab & complement cascade, reduction of MHC class II expression & $(61-63)$ \\
Proteasome Inhibition & Depletion of circulating B-cells & $(10,64)$ \\
Complement Inhibition & Plasma cell apoptosis & Inhibition of formation of the membrane attack complex \\
\hline
\end{tabular}

AMR, antibody-mediated rejection; DSA, donor-specific antibodies.

immunodeficiency syndromes and passive immunity but also has applications in immune mediated conditions such as Guillan-Barré syndrome, immune thrombocytopenia (ITP), and myasthenia gravis. IVIG has become a standard part of protocols for treatment of AMR, but the precise mechanism of action is unclear (57). Proposed mechanisms include modulation of expression and function of the Fcy receptor, inactivation of DSA, downregulation of B-cells, inhibition of the complement cascade and the cytokine network, and reduction of MHC class II antigen expression $(57,58)$. IVIG may be used as monotherapy but is more commonly used in conjunction with plasmapheresis and/or corticosteroids $(57,59)$. Dosing of IVIG has been variable with ranges from $100-2,000 \mathrm{mg} / \mathrm{kg}(4,57)$.

\section{Rituximab}

Rituximab is an anti-CD20 antibody that binds to preB-cells and mature B-lymphocytes in circulation, lymph nodes, and bone marrow and induces apoptosis and cell lysis (60). While rituximab depletes circulating B-cells, it has no known effect on plasma cells that may already be producing antibody. Rituximab has been studied in the treatment of B-cell neoplasms and autoimmune conditions (60). Rituximab plus IVIG was found to improve survival over IVIG alone in a protocol to preemptively treat DSA; however, groups were not randomized and no difference in rejection rates was found (37). Indeed, data for the treatment of AMR with rituximab are sparse.

\section{Proteasome inbibition}

Proteasomes degrade misfolded and ubiquitinated proteins.
Bortezomib and carfilzomib bind and inhibit proteasomes in plasma cells allowing the accumulation of these proteins and leading to plasma cell apoptosis (61-63). Proteasome inhibitors have largely been studied in the treatment of multiple myeloma $(61,62)$. Observational studies have shown that treatment with proteasome inhibitors in AMR depletes DSA and improves allograft function $(61,62)$, but no head-to-head comparisons or clinical trials have been published to date.

\section{Complement inbibition}

Eculizumab is a monoclonal antibody that binds to C5 and inhibits formation of the MAC. Eculizumab has largely been studied in atypical hemolytic uremic syndrome. Case reports utilizing eculizumab as part of a multimodal treatment regimen for AMR have shown depletion of DSA and improvement in allograft function $(10,64)$, but no headto-head comparisons or clinical trials have been published to date.

\section{Long-term outcomes}

Despite improvements in diagnosis, clinical outcomes after AMR remain poor (39). Astor and colleagues reported a case series of 40 patients who developed AMR and found 1 - and 5 -year survival to be near $80 \%$ and $40 \%$ respectively (46). Otani and colleagues presented a series of 9 patients who received therapy for AMR in which 5/9 initially improved with therapy, but 7/9 eventually developed CLAD and 6/9 (67\%) died within 3 years of diagnosis of AMR (4). Witt and colleagues reported a series 
of 21 patients who developed AMR of whom 6/21 died of refractory AMR, and all 15 who improved eventually developed CLAD (3). Overall, 15/21 (71\%) patients died during the study period and the median survival after the diagnosis of AMR was 593 days (3). Aguilar and colleagues reported a series of 73 cases of AMR and found a 30-day mortality of $26 \%$ despite aggressive antibody depleting treatment (52). Indeed, multiple reports have shown that the inability to clear DSA and a diagnosis of AMR are associated with increased risk of CLAD $(3,4)$. These reports suggest that although AMR may be a reversible cause of acute graft dysfunction, there is a high incidence of CLAD and poor long-term survival after AMR.

\section{Sensitization prior to transplantation}

The presence of HLA antibodies prior to transplantation has been associated with variable outcomes. While some reports found no difference in survival, acute rejection, or BOS, others have shown decreased survival, decreased rates of transplantation, prolonged waiting times, and increases in post-transplant ventilator days, acute rejection, AMR, and BOS (65-70). Notably, HLA class II antibodies have been associated with worse outcomes $(3,52,68)$. Brugiere and colleagues found that patients with class II DSA had significantly higher mortality and significantly lower freedom from BOS (68). Descriptive studies by Witt et al. and Aguilar et al. also noted an association between HLA class II antibodies, especially HLA-DQ antibodies, and the development of AMR $(3,52)$. Notably, pro-inflammatory cytokines have been shown to induce the expression of class II HLA on pulmonary endothelial cells $(26,27)$. Together, these studies illustrate that endothelial cells are the focal point of antibody-mediated injury.

Desensitization protocols have been implemented for sensitized patients with variable success. Appel and colleagues treated 12/35 (34\%) sensitized patients with peri-transplant IVIG and extracorporeal immunoadsorption (ECI) and found a significant decrease in acute rejection but no significant difference in survival or BOS (71). Snyder and colleagues treated 18/114 (16\%) sensitized patients with a desensitization protocol including plasmapheresis, methylprednisolone, bortezomib, rituximab, and IVIG (72). Among the 9/18 (50\%) treated patients who went on to receive a transplant, they found a significant decrease in HLA antibody with pretreatment MFI 5,000-10,000; however, they found no difference in PRA, cPRA, or post-transplant survival (72). Tinckam and colleagues treated 146 patients with known DSA or with PRA $>30 \%$ with a desensitization protocol including perioperative plasma exchange, IVIG, anti-thymocyte globulin, and mycophenolic acid and compared them to 194 unsensitized controls (73). They found that treated, sensitized recipients had significantly lower rates of acute rejection compared with unsensitized controls and found no significant difference in spirometry or 1-year graft survival (73). Courtwright and colleagues reviewed 203 cases, 18 of whom had pretransplant DSA and were treated with peri-transplant plasmapheresis, and found that sensitized patients were more likely to require prolonged mechanical ventilation and to have AMR (74). They found no difference in mortality or CLAD-free survival between groups (74). While some centers have shown success, small sample size, varied protocols and immunosuppressive regimens, and heterogenous patient populations make it challenging to draw conclusions regarding the role and impact of desensitization protocols.

\section{Gaps in knowledge and future directions}

Despite the advances in diagnostic criteria and treatment modalities in AMR, significant gaps in knowledge remain. The true incidence of AMR remains unknown with prior reports describing widely variable incidence from $4 \%$ to greater than $50 \%(3,37,75,76)$. The previous lack of consensus diagnostic criteria and highly sensitive assays for DSA may explain this variability. However, the limitations of the current consensus diagnostic criteria present further challenges in defining the true incidence of AMR. In addition, chronic and subclinical AMR are illdefined, and the impact of these potential forms of rejection on long-term outcomes remains unknown. The lack of highly sensitive assays for non-HLA antibodies further limits our understanding of AMR. While inability to clear DSA is associated with CLAD and poor survival, the associated mechanisms remain unclear, and protocols for prevention and treatment have yet to be developed. Longterm outcomes after AMR are poor and data for specific treatment protocols remain sparse. Large, multicenter, randomized clinical trials are necessary to better define the optimal treatment regimen. These studies need not be placebo controlled as equipoise exists for head-to-head comparisons of previously studied regimens. Furthermore, dosing studies are necessary to identify the optimal doses for treatment of AMR as current dosing strategies are based on the use of agents in other medical conditions. 
Finally, as investigations of new diagnostic techniques and treatment strategies emerge, large, multicenter studies will be necessary to identify best-practices for implementation.

There are multiple areas of ongoing and future investigation in AMR including in the areas of mechanisms, diagnosis, and treatment. Li and colleagues developed a mouse lung re-transplantation model of AMR and found that tolerant allografts developed BALT and that depletion of regulatory T-cells led to AMR (38). They suggest a role for future investigation of the pathogenesis of AMR utilizing this model and the treatment of AMR by costimulatory blockade of T-cells and B-cells (38).

Given the limitations of the current consensus diagnostic criteria for AMR, alternative methods for diagnosis are actively being investigated including donor-derived cell-free DNA (ddcfDNA) and transcriptome analyses. The presence of injury in the allograft results in donor cell apoptosis and circulation of donor DNA in the serum of recipients (77-80). Assays have been developed that can quantify the amount of ddcfDNA in recipient serum (77-81). This technique involves sequencing DNA found in the serum of recipients and differentiating and quantifying donor DNA $(77,81)$. These assays were found to have excellent sensitivity for acute rejection, but specificity is poor (77-81). Notably, Agbor-Enoh and colleagues found the sensitivity for AMR to be approaching $100 \%$, but specificity was only $35 \%$; however, when coupled with testing for DSA, specificity improved to $90 \%(82,83)$. In addition, they found that elevations in ddcfDNA preceded the clinical diagnosis of AMR by a median of 2.8 months (82). Measurement of cellfree DNA could serve as a noninvasive method to diagnose impending AMR; however, it is unclear if treatment of impending AMR will improve outcomes. The Early Detection and Treatment of AMR (eDATA) study proposes to identify patients who have elevated ddcfDNA and DSA and to initiate treatment prior to developing clinical AMR. This study is currently in progress and will compare outcomes with conventional diagnosis and treatment of clinical AMR (83).

Another area of active investigation is transcriptome analysis. Micro RNAs (miRNA) are short, noncoding RNA sequences that regulate gene expression. Transcriptome analysis can be performed on BAL cell pellets, peripheral blood mononuclear cells, and biopsy specimens (84-88). Early studies have shown that differential expression of miRNAs correlate with episodes of acute rejection and may even precede diagnosis of chronic rejection (84-88). While early data for antibody associated chronic rejection exists (84), transcriptome analysis has yet to be evaluated in the diagnosis of AMR.

Valenzuela and colleagues reviewed emerging therapies for AMR that inhibit antibody formation and antibodymediated allograft injury by targeting CTLA4, IL-6 receptor, B-cell activating factor, $\mathrm{C} 1, \mathrm{mTOR}$, and the Fc region of bound antibody (40). Future studies should evaluate the role of these alternate pathways of immune modulation in AMR.

\section{Conclusions}

AMR generally results in severe acute allograft dysfunction and is associated with increased risk of CLAD and poor survival. Despite development of consensus diagnostic criteria, treatment protocols have limited data and longterm outcomes remain poor. Promising diagnostic techniques are actively being investigated that may allow for early detection and treatment of AMR. Further research is required to identify and define chronic and subclinical AMR, and head-to-head comparisons of currently used treatment protocols are necessary to identify an optimal treatment approach. Gaps in knowledge regarding the epidemiology, mechanisms, diagnosis, and treatment of AMR persist and future research should focus on addressing these gaps.

\section{Acknowledgments}

Funding: None.

\section{Footnote}

Provenance and Peer Review: This article was commissioned by the Guest Editors (Masaaki Sato and Dong Tian) for the series "Strategies to Achieve Long-Term Success of Lung Transplantation" published in Annals of Translational Medicine. The article was sent for external peer review organized by the Guest Editors and the editorial office.

Conflicts of Interest: The series "Strategies to Achieve LongTerm Success of Lung Transplantation" was commissioned by the editorial office without any funding or sponsorship. The authors have no conflicts of interest to declare.

Ethical Statement: The authors are accountable for all aspects of the work in ensuring that questions related to the accuracy or integrity of any part of the work are 
appropriately investigated and resolved.

Open Access Statement: This is an Open Access article distributed in accordance with the Creative Commons Attribution-NonCommercial-NoDerivs 4.0 International License (CC BY-NC-ND 4.0), which permits the noncommercial replication and distribution of the article with the strict proviso that no changes or edits are made and the original work is properly cited (including links to both the formal publication through the relevant DOI and the license). See: https://creativecommons.org/licenses/by-nc-nd/4.0/.

\section{References}

1. Chambers DC, Cherikh WS, Goldfarb SB, et al. The International Thoracic Organ Transplant Registry of the International Society for Heart and Lung Transplantation: Thirty-fifth adult lung and heart-lung transplant report-2018; Focus theme: Multiorgan Transplantation. J Heart Lung Transplant 2018;37:1169-83.

2. Lobo LJ, Aris RM, Schmitz J, et al. Donor-specific antibodies are associated with antibody-mediated rejection, acute cellular rejection, bronchiolitis obliterans syndrome, and cystic fibrosis after lung transplantation. J Heart Lung Transplant 2013;32:70-7.

3. Witt CA, Gaut JP, Yusen RD, et al. Acute antibodymediated rejection after lung transplantation. J Heart Lung Transplant 2013;32:1034-40.

4. Otani S, Davis AK, Cantwell L, et al. Evolving experience of treating antibody-mediated rejection following lung transplantation. Transpl Immunol 2014;31:75-80.

5. Roux A, Bendib Le Lan I, Holifanjaniaina S, et al. Antibody-Mediated Rejection in Lung Transplantation: Clinical Outcomes and Donor-Specific Antibody Characteristics. Am J Transplant 2016;16:1216-28.

6. Levine DJ, Glanville AR, Aboyoun C, et al. Antibodymediated rejection of the lung: A consensus report of the International Society for Heart and Lung Transplantation. J Heart Lung Transplant 2016;35:397-406.

7. Morrell MR, Patterson GA, Trulock EP, et al. Acute antibody-mediated rejection after lung transplantation. J Heart Lung Transplant 2009;28:96-100.

8. Frost AE, Jammal CT, Cagle PT. Hyperacute rejection following lung transplantation. Chest 1996;110:559-62.

9. Kulkarni HS, Bemiss BC, Hachem RR. Antibody-mediated Rejection in Lung Transplantation. Curr Transplant Rep 2015;2:316-23.

10. Dawson KL, Parulekar A, Seethamraju H. Treatment of hyperacute antibody-mediated lung allograft rejection with eculizumab. J Heart Lung Transplant 2012;31:1325-6.

11. Hachem R. Antibody-Mediated Lung Transplant Rejection. Curr Respir Care Rep 2012;1:157-61.

12. Hadjiliadis D, Chaparro C, Reinsmoen NL, et al. Pretransplant panel reactive antibody in lung transplant recipients is associated with significantly worse posttransplant survival in a multicenter study. J Heart Lung Transplant 2005;24:S249-54.

13. Bosanquet JP, Witt CA, Bemiss BC, et al. The impact of pre-transplant allosensitization on outcomes after lung transplantation. J Heart Lung Transplant 2015;34:1415-22.

14. Badesch DB, Zamora M, Fullerton D, et al. Pulmonary capillaritis: a possible histologic form of acute pulmonary allograft rejection. J Heart Lung Transplant 1998;17:415-22.

15. Magro CM, Deng A, Pope-Harman A, et al. Humorally mediated posttransplantation septal capillary injury syndrome as a common form of pulmonary allograft rejection: a hypothesis. Transplantation 2002;74:1273-80.

16. Djamali A, Kaufman DB, Ellis TM, et al. Diagnosis and management of antibody-mediated rejection: current status and novel approaches. Am J Transplant 2014;14:255-71.

17. Takemoto SK, Zeevi A, Feng S, et al. National conference to assess antibody-mediated rejection in solid organ transplantation. Am J Transplant 2004;4:1033-41.

18. Farkash EA, Colvin RB. Diagnostic challenges in chronic antibody-mediated rejection. Nat Rev Nephrol 2012;8:255-7.

19. Hidalgo LG, Sis B, Sellares J, et al. NK cell transcripts and NK cells in kidney biopsies from patients with donorspecific antibodies: evidence for NK cell involvement in antibody-mediated rejection. Am J Transplant 2010;10:1812-22.

20. Sis B, Halloran PF. Endothelial transcripts uncover a previously unknown phenotype: $\mathrm{C} 4 \mathrm{~d}$-negative antibodymediated rejection. Curr Opin Organ Transplant 2010;15:42-8.

21. Patel R, Terasaki PI. Significance of the positive crossmatch test in kidney transplantation. $\mathrm{N}$ Engl J Med 1969;280:735-9.

22. Stiller CR, Sinclair NR, Abrahams S, et al. Lymphocytedependent antibody and renal graft rejection. Lancet 1975;1:953-4.

23. Kissmeyer-Nielsen F, Olsen S, Petersen VP, et al. Hyperacute rejection of kidney allografts, associated with pre-existing humoral antibodies against donor cells. Lancet 1966;2:662-5.

24. Hachem RR, Kamoun M, Budev MM, et al. Human 
leukocyte antigens antibodies after lung transplantation: Primary results of the HALT study. Am J Transplant 2018;18:2285-94.

25. Angaswamy N, Tiriveedhi V, Sarma NJ, et al. Interplay between immune responses to HLA and non-HLA self-antigens in allograft rejection. Hum Immunol 2013;74:1478-85.

26. Cunningham AC, Zhang JG, Moy JV, et al. A comparison of the antigen-presenting capabilities of class II MHCexpressing human lung epithelial and endothelial cells. Immunology 1997;91:458-63.

27. Shreeniwas R, Schulman LL, Narasimhan M, et al. Adhesion molecules (E-selectin and ICAM-1) in pulmonary allograft rejection. Chest 1996;110:1143-9.

28. Bharat A, Subramaniam V, Kreisel D, et al. Novel TwoHit Hypothesis for the Development of De Novo Autoimmunity Following Lung Transplantation. The Journal of Heart and Lung Transplantation 2014;33:S81.

29. Loupy A, Lefaucheur C, Vernerey D, et al. Complementbinding anti-HLA antibodies and kidney-allograft survival. N Engl J Med 2013;369:1215-26.

30. Zeevi A, Lunz J, Feingold B, et al. Persistent strong anti-HLA antibody at high titer is complement binding and associated with increased risk of antibody-mediated rejection in heart transplant recipients. J Heart Lung Transplant 2013;32:98-105.

31. Valenzuela NM, McNamara JT, Reed EF. Antibodymediated graft injury: complement-dependent and complement-independent mechanisms. Curr Opin Organ Transplant 2014;19:33-40.

32. Kuo HH, Morrell CN, Baldwin WM 3rd. Alloantibody induced platelet responses in transplants: potent mediators in small packages. Hum Immunol 2012;73:1233-8.

33. Sis B. Endothelial molecules decipher the mechanisms and functional pathways in antibody-mediated rejection. Hum Immunol 2012;73:1218-25.

34. Trayssac M, Negre-Salvayre A, Thomsen M. Mechanisms of human smooth muscle cell proliferation and transplant vasculopathy induced by HLA class I antibodies: in vitro and in vivo studies. Hum Immunol 2012;73:1253-60.

35. Zhang X, Valenzuela NM, Reed EF. HLA class I antibodymediated endothelial and smooth muscle cell activation. Curr Opin Organ Transplant 2012;17:446-51.

36. Jaramillo A, Smith CR, Maruyama T, et al. Anti-HLA class I antibody binding to airway epithelial cells induces production of fibrogenic growth factors and apoptotic cell death: a possible mechanism for bronchiolitis obliterans syndrome. Hum Immunol 2003;64:521-9.
37. Hachem RR, Yusen RD, Meyers BF, et al. Anti-human leukocyte antigen antibodies and preemptive antibodydirected therapy after lung transplantation. J Heart Lung Transplant 2010;29:973-80.

38. Li W, Gauthier JM, Higashikubo R, et al. Bronchusassociated lymphoid tissue-resident Foxp3 + T lymphocytes prevent antibody-mediated lung rejection. J Clin Invest 2019;129:556-68.

39. Hachem RR. Acute Rejection and Antibody-Mediated Rejection in Lung Transplantation. Clin Chest Med 2017;38:667-75.

40. Valenzuela NM, Reed EF. Antibody-mediated rejection across solid organ transplants: manifestations, mechanisms, and therapies. J Clin Invest 2017;127:2492-504.

41. DeNicola MM, Weigt SS, Belperio JA, et al. Pathologic findings in lung allografts with anti-HLA antibodies. J Heart Lung Transplant 2013;32:326-32.

42. Yousem SA, Zeevi A. The histopathology of lung allograft dysfunction associated with the development of donor-specific HLA alloantibodies. Am J Surg Pathol 2012;36:987-92.

43. Tambur AR, Herrera ND, Haarberg KM, et al. Assessing Antibody Strength: Comparison of MFI, C1q, and Titer Information. Am J Transplant 2015;15:2421-30.

44. Roden AC, Aisner DL, Allen TC, et al. Diagnosis of Acute Cellular Rejection and Antibody-Mediated Rejection on Lung Transplant Biopsies: A Perspective From Members of the Pulmonary Pathology Society. Arch Pathol Lab Med 2017;141:437-44.

45. Roux A, Levine DJ, Zeevi A, et al. Banff Lung Report: Current knowledge and future research perspectives for diagnosis and treatment of pulmonary antibody-mediated rejection (AMR). Am J Transplant 2019;19:21-31.

46. Astor TL, Weill D, Cool C, et al. Pulmonary capillaritis in lung transplant recipients: treatment and effect on allograft function. J Heart Lung Transplant 2005;24:2091-7.

47. Berry G, Burke M, Andersen C, et al. Pathology of pulmonary antibody-mediated rejection: 2012 update from the Pathology Council of the ISHLT. J Heart Lung Transplant 2013;32:14-21.

48. Stewart S, Fishbein MC, Snell GI, et al. Revision of the 1996 working formulation for the standardization of nomenclature in the diagnosis of lung rejection. J Heart Lung Transplant 2007;26:1229-42.

49. Wallace WD, Weigt SS, Farver CF. Update on pathology of antibody-mediated rejection in the lung allograft. Curr Opin Organ Transplant 2014;19:303-8.

50. Roden AC, Maleszewski JJ, Yi ES, et al. Reproducibility of 
Complement 4d deposition by immunofluorescence and immunohistochemistry in lung allograft biopsies. J Heart Lung Transplant 2014;33:1223-32.

51. González-Molina M, Ruiz-Esteban P, Caballero A, et al. Immune response and histology of humoral rejection in kidney transplantation. Nefrologia 2016;36:354-67.

52. Aguilar PR, Carpenter D, Ritter J, et al. The role of C4d deposition in the diagnosis of antibody-mediated rejection after lung transplantation. Am J Transplant 2018;18:936-44.

53. Coutinho AE, Chapman KE. The anti-inflammatory and immunosuppressive effects of glucocorticoids, recent developments and mechanistic insights. Mol Cell Endocrinol 2011;335:2-13.

54. Chih S, Tinckam KJ, Ross HJ. A survey of current practice for antibody-mediated rejection in heart transplantation. Am J Transplant 2013;13:1069-74.

55. Roberts DM, Jiang SH, Chadban SJ. The treatment of acute antibody-mediated rejection in kidney transplant recipients-a systematic review. Transplantation 2012;94:775-83.

56. Bittner HB, Dunitz J, Hertz M, et al. Hyperacute rejection in single lung transplantation--case report of successful management by means of plasmapheresis and antithymocyte globulin treatment. Transplantation 2001;71:649-51.

57. Jordan SC, Toyoda M, Kahwaji J, et al. Clinical aspects of intravenous immunoglobulin use in solid organ transplant recipients. Am J Transplant 2011;11:196-202.

58. Durandy A, Kaveri SV, Kuijpers TW, et al. Intravenous immunoglobulins--understanding properties and mechanisms. Clin Exp Immunol 2009;158 Suppl 1:2-13.

59. Shehata N, Palda VA, Meyer RM, et al. The use of immunoglobulin therapy for patients undergoing solid organ transplantation: an evidence-based practice guideline. Transfus Med Rev 2010;24 Suppl 1:S7-27.

60. Golay J, Semenzato G, Rambaldi A, et al. Lessons for the clinic from rituximab pharmacokinetics and pharmacodynamics. MAbs 2013;5:826-37.

61. Ensor CR, Yousem SA, Marrari M, et al. Proteasome Inhibitor Carfilzomib-Based Therapy for AntibodyMediated Rejection of the Pulmonary Allograft: Use and Short-Term Findings. Am J Transplant 2017;17:1380-8.

62. Stuckey LJ, Kamoun M, Chan KM. Lung transplantation across donor-specific anti-human leukocyte antigen antibodies: utility of bortezomib therapy in early graft dysfunction. Ann Pharmacother 2012;46:e2.

63. Adams J, Kauffman M. Development of the proteasome inhibitor Velcade (Bortezomib). Cancer Invest 2004;22:304-11.

64. Muller YD, Aubert JD, Vionnet J, et al. Acute Antibodymediated Rejection 1 Week After Lung Transplantation Successfully Treated With Eculizumab, Intravenous Immunoglobulins, and Rituximab. Transplantation 2018;102:e301-3.

65. Gammie JS, Pham SM, Colson YL, et al. Influence of panel-reactive antibody on survival and rejection after lung transplantation. J Heart Lung Transplant 1997;16:408-15.

66. Lau CL, Palmer SM, Posther KE, et al. Influence of panelreactive antibodies on posttransplant outcomes in lung transplant recipients. Ann Thorac Surg 2000;69:1520-4.

67. Kim M, Townsend KR, Wood IG, et al. Impact of pretransplant anti-HLA antibodies on outcomes in lung transplant candidates. Am J Respir Crit Care Med 2014;189:1234-9.

68. Brugière $\mathrm{O}$, Suberbielle $\mathrm{C}$, Thabut $\mathrm{G}$, et al. Lung transplantation in patients with pretransplantation donor-specific antibodies detected by Luminex assay. Transplantation 2013;95:761-5.

69. Smith JD, Ibrahim MW, Newell H, et al. Pre-transplant donor HLA-specific antibodies: characteristics causing detrimental effects on survival after lung transplantation. J Heart Lung Transplant 2014;33:1074-82.

70. Zazueta OE, Preston SE, Moniodis A, et al. The Presence of Pretransplant HLA Antibodies Does Not Impact the Development of Chronic Lung Allograft Dysfunction or CLAD-Related Death. Transplantation 2017;101:2207-12.

71. Appel JZ 3rd, Hartwig MG, Davis RD, et al. Utility of peritransplant and rescue intravenous immunoglobulin and extracorporeal immunoadsorption in lung transplant recipients sensitized to HLA antigens. Hum Immunol 2005;66:378-86.

72. Snyder LD, Gray AL, Reynolds JM, et al. Antibody desensitization therapy in highly sensitized lung transplant candidates. Am J Transplant 2014;14:849-56.

73. Tinckam KJ, Keshavjee S, Chaparro C, et al. Survival in sensitized lung transplant recipients with perioperative desensitization. Am J Transplant 2015;15:417-26.

74. Courtwright AM, Cao S, Wood I, et al. Clinical Outcomes of Lung Transplantation in the Presence of Donor-Specific Antibodies. Ann Am Thorac Soc 2019;16:1131-7.

75. Girnita AL, McCurry KR, Iacono AT, et al. HLA-specific antibodies are associated with high-grade and persistentrecurrent lung allograft acute rejection. J Heart Lung Transplant 2004;23:1135-41.

76. Snyder LD, Wang Z, Chen DF, et al. Implications 
for human leukocyte antigen antibodies after lung transplantation: a 10-year experience in 441 patients. Chest 2013;144:226-33.

77. De Vlaminck I, Martin L, Kertesz M, et al. Noninvasive monitoring of infection and rejection after lung transplantation. Proc Natl Acad Sci U S A 2015;112:13336-41.

78. Agbor-Enoh S, Wang Y, Tunc I, et al. Donor-derived cellfree DNA predicts allograft failure and mortality after lung transplantation. EBioMedicine 2019;40:541-53.

79. Tanaka S, Sugimoto S, Kurosaki T, et al. Donor-derived cell-free DNA is associated with acute rejection and decreased oxygenation in primary graft dysfunction after living donor-lobar lung transplantation. Sci Rep 2018;8:15366.

80. Yang JYC, Verleden SE, Zarinsefat A, et al. Cell-Free DNA and CXCL10 Derived from Bronchoalveolar Lavage Predict Lung Transplant Survival. J Clin Med 2019. doi: 10.3390/jcm8020241.

81. Zou J, Duffy B, Slade M, et al. Rapid detection of donor cell free DNA in lung transplant recipients with rejections using donor-recipient HLA mismatch. Hum Immunol 2017;78:342-9.

82. Agbor-Enoh S, Jackson AM, Tunc I, et al. Late manifestation of alloantibody-associated injury and clinical pulmonary antibody-mediated rejection: Evidence from cell-free DNA analysis. J Heart Lung Transplant 2018;37:925-32.

83. Agbor-Enoh S. 2018 ATS BEAR Cage Winning Proposal: Cell-Free DNA to Improve Lung Transplant Outcomes. Am J Respir Crit Care Med 2019;199:1058-60.

84. Nayak DK, Zhou F, Xu M, et al. Zbtb7a induction in alveolar macrophages is implicated in anti-HLA-mediated lung allograft rejection. Sci Transl Med 2017. doi: 10.1126/scitranslmed.aal1243.

85. Weigt SS, Wang X, Palchevskiy V, et al. Gene Expression Profiling of Bronchoalveolar Lavage Cells Preceding a Clinical Diagnosis of Chronic Lung Allograft Dysfunction. PLoS One 2017;12:e0169894.

86. Weigt SS, Wang X, Palchevskiy V, et al. Usefulness of gene expression profiling of bronchoalveolar lavage cells in acute lung allograft rejection. J Heart Lung Transplant 2019;38:845-55.

87. Zhang $W$, Zhou T, Ma SF, et al. MicroRNAs Implicated in Dysregulation of Gene Expression Following Human Lung Transplantation. Transl Respir Med 2013. doi: 10.1186/2213-0802-1-12.

88. Xu Z, Nayak DK, Benshoff N, et al. De novo-developed antibodies to donor MHC antigens lead to dysregulation of microRNAs and induction of MHC class II. J Immunol 2015;194:6133-43.
Cite this article as: Bery AI, Hachem RR. Antibodymediated rejection after lung transplantation. Ann Transl Med 2020;8(6):411. doi: 10.21037/atm.2019.11.86 